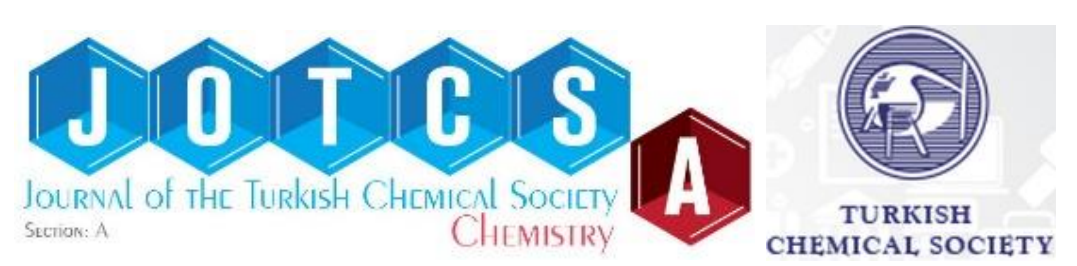

\title{
A Sensitive Quantification of Agmatine Using a Hybrid Electrode Based on Zinc Oxide Nanoparticles
}

\author{
Hilal İNCEBAY1 1 \\ ${ }^{1}$ University of Nevsehir Hacı Bektas Veli, 50300, Nevsehir, Turkey.
}

Abstract: An electrochemical sensor was prepared by modifiying a hybrid of multi-walled carbon nanotubes (MWCNTs) and zinc oxide nanoparticles (ZnONPs) to a glassy carbon (GC) electrode surface to accurately determine agmatine. The ZnONPs+MWCNTs/GC electrode surface was characterized using scanning electron microscopy (SEM) and energy dispersive X-ray (XRD). Agmatine did not exhibit any peak on the GC electrode surface, but exhibited a large oxidation peak at $637.9 \mathrm{mV}$ on the MWCNTs/GC electrode surface. Furthermore, it was observed that the electrochemical behavior of agmatine was greatly improved on the MWCNT+ZnONPs/GC electrode surface and that this surface exhibited a well-defined higher current peak at $581.9 \mathrm{mV}$. The electrochemical responses of agmatine on the MWCNT+ZnONPs/GC electrode surface were performed using square-wave voltammetry (SWV). A linear plot was obtained for the current responses of agmatine against concentrations in the range of $0.1 \mu \mathrm{M}-5.2 \mu \mathrm{M}$ yielding a detection limit of $4.13 \times 10^{-8} \mathrm{M}$ (based on $3 \mathrm{~S}_{\mathrm{b}} / \mathrm{m}$ ). The accurate quantification of agmatine makes the ZnONPs+MWCNTs/GC electrode system of great interest for the treatment of schizophrenia.

Keywords: Agmatine, zinc oxide nanoparticles, hybrid, sensor.

Submitted:March 05, 2018. Accepted: October 17, 2018.

Cite This: İncebay H. A Sensitive Quantification of Agmatine Using a Hybrid Electrode Based on Zinc Oxide Nanoparticles. JOTCSA. 2018; 5(3):1205-14.

DOI: http://dx.doi.org/10.18596/jotcsa.401450.

Corresponding Author. E-mail: hilalincebay@gmail.com

\section{INTRODUCTION}

Agmatine is a cationic polyamine produced naturally by the decarboxylation of $\mathrm{L}$-arginine by arginine decarboxylase enzyme. It is produced in the mammalian brain and is also preserved in nature $(1,2)$. Agmatine has the properties of a neurotransmitter synthesized in the brain, stored in the vesicles and released from the brain (3-5). Current studies demonstrate that agmatine meets all the necessary criteria for the definition of a neurotransmitter and has been accepted as a new neurotransmitter (6-8). In addition, many studies have shown that agmatine degradation products such as spermidine and spermine are found at high rates in the brains, cerebrospinal fluid, and blood of patients with schizophrenia. The high levels of these metabolites in patients with schizophrenia have led to the idea that agmatine may be present at high concentrations (9). As a matter of fact, a study published by Uzbay et al. found that the levels of agmatine in patients with schizophrenia who did not take any medication for at least 6 months were significantly higher than those of healthy controls (10). There are studies that suggest that there may be a relationship between schizophrenia and increased agmatine concentrations due to excessive release of agmatine and agmatine derivatives in the brain and cerebrospinal fluid $(2,11,12)$. For this reason, it is important to determine agmatine levels correctly and quickly. Highperformance liquid chromatography methods are generally used for agmatine measurements. However, due to problems such as selectivity and time-consuming steps 
for derivatization, these techniques can create problems in conducting correctly analysis of samples. Nevertheless, voltammetric techniques with modified electrodes provide advantages such as high selectivity, speed, and simplicity. Furthermore, voltammetric methods have attracted attention in the analysis of many samples since they have the properties of speed, selectivity, and reproducibility $(13,14)$. The preparation of electrode surfaces in the nanostructure has also attracted focus, particularly regarding the design of electrochemical sensing electrodes (15-17). Of these nanostructures, carbon nanotubes (CNTs), which have excellent electrocatalytic activity and sensitivity, are applied to obtain electrochemical sensors and biosensors. In addition, electrodes modified with hybrids of nanoparticles of CNTs and metal oxides offer good performance benefits, such as increased sensitivity, reduced overvoltage, mass transport, catalysis, and a high effective surface area and detection limit (18-19).

$\mathrm{HN}$

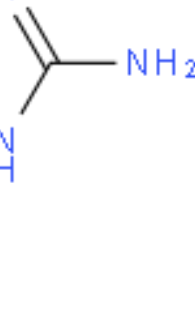

Figure 1. Chemical structure of agmatine.

In this study, a GC electrode surface was modified with a hybrid of MWCNTs and ZnO nanoparticles for agmatine quantification. The modification of the GC electrode surface with this hybrid provided excellent electrocatalytic efficiency, good selectivity and high sensitivity.

\section{EXPERIMENTAL}

\section{Chemical reagents}

Agmatine, multi-walled carbon nanotubes (MWCNTs), zinc oxide nanoparticles (ZnONPs), sodium hydroxide $(\mathrm{NaOH})$ and disodium phosphate dibasic $\left(\mathrm{Na}_{2} \mathrm{HPO}_{4}\right)$ were purchased from Sigma (Missouri, USA). Acetonitrile and chloroform were obtained from VWR International (Radnor, USA). Potassium dihydrogen phosphate $\left(\mathrm{KH}_{2} \mathrm{PO}_{4}\right)$ was purchased from Merck (Darmstadt, Germany). The solutions were prepared with ultrapure water.

\section{Instrumentation}

A Gamry Interface 1000B Potentiostat/Galvanostat/ZRA was used to apply electrochemical measurements with cyclic voltammetry (CV) and square-wave voltammetry (SWV) techniques. Electrochemical measurements were performed in a three-electrode cell. A glassy carbon (GC) electrode was used as working electrode (MF-2012 BASi, USA), $\mathrm{Ag} / \mathrm{AgCl} /\left(\mathrm{KCl}_{\text {sat. }}\right)$ was used as the reference electrode (MF-2052 BASi, USA), and Pt wire was used as the counter electrode. All experimental data was plotted with the Origin
8.0 program so that a comparison between the data was made.

\section{Preparation of modified electrodes}

GC electrodes were first polished with $0.05 \mu \mathrm{m}$ and $0.30 \mu \mathrm{m}$ alumina slurry on a velvet cleaning pad and then the electrodes were sonicated for 5 minutes in pure water and acetonitrile. Then commercial MWCNTs were sonicated in concentrated $\mathrm{HClO}_{4}+\mathrm{HNO}_{3}(3: 7$, $\mathrm{v}: \mathrm{v})$ for 5 hours to functionalize the MWCNTs surfaces (20). The functionalized MWCNTs were washed with ultrapure water and dried. MWCNTs, ZnONPs, and ZnONPs+MWCNTs suspensions were then prepared by sonicating $1.0 \mathrm{mg}$ of functionalized MWCNTs, $0.1 \mathrm{mg}$ of ZnONPs and $0.1 \mathrm{mg}$ of ZnONPs plus $1.0 \mathrm{mg}$ of functionalized MWCNTs in $5 \mathrm{~mL}$ of chloroform for $50 \mathrm{~min}$. MWCNTs/GC, ZnONPs/GC and ZnONPs+MWCNTs/GC electrode surfaces were prepared by dropping $5 \mu \mathrm{L}$ of each of the prepared suspensions onto cleaned GC electrode surfaces. Afterward, the surfaces of these electrodes were extensively washed with ultra-pure water.

\section{Optimization of Modified Electrode}

The experimental results showed that the best results were obtained by sonicating MWCNTs and ZnONPs mixture in $5 \mathrm{~mL}$ chloroform at a 10:1 ratio for 50 minutes. On the other hand, the composite layer on GC electrode surface showed a clear peak formation for agmatine when the amount of ZnONPs/MWCNTs suspension was $5 \mu \mathrm{L}$. Besides, a considerable decrease in sensitivity and reproducibility was observed when higher amounts of this 
suspension were added to the GC electrode surface.

\section{Voltammetric analysis of agmatine}

The prepared ZnONPs+MWCNTs/GC electrode was immersed in a cell containing agmatine with a concentration of $1.0 \times 10^{-7} \mathrm{M}$ dissolved in $50 \mathrm{mM}$ phosphate buffer solution (PBS) $(\mathrm{pH}$ 7.0) and the voltammogram was recorded with CV. Under the same conditions, voltammograms of agmatine were taken at the bare/GC and MWCNTs/GC electrode surfaces and compared with the ZnONPs+MWCNTs/GC electrode. Then, voltammograms were taken at different scan rates for the ZnONPs+MWCNTs/GC electrode surface at the maximum voltammetric response to accurately conceptualize the electrochemical process of agmatine. Voltammograms of agmatine at the ZnONPs+MWCNTs/GC electrode surface were then taken at various $\mathrm{pH}$ values to determine the $\mathrm{pH}$ level at which the best voltammetric response was observed for agmatine
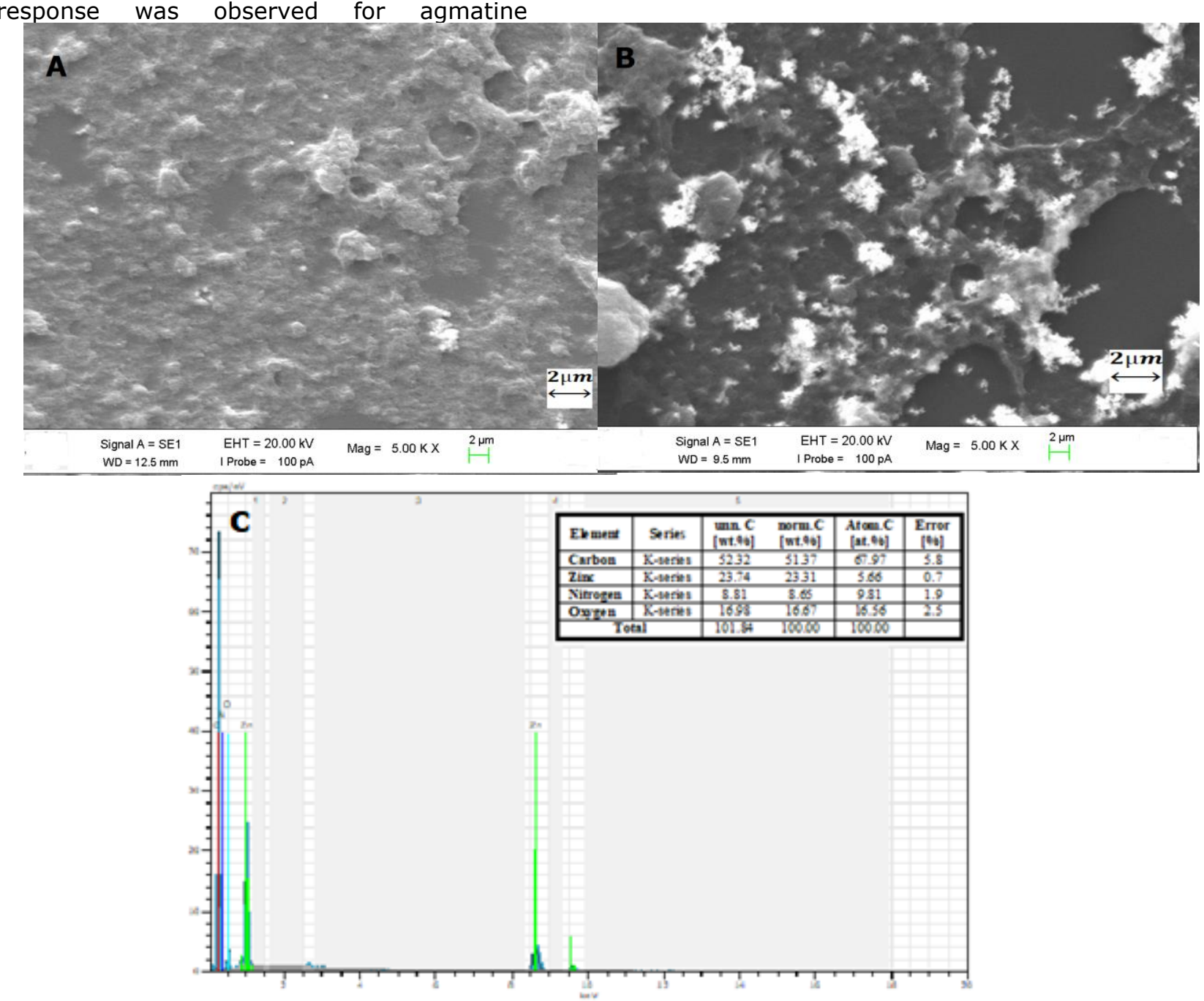

Figure 2. SEM images of A) MWCNTS/GC electrode surface, B) ZnONPs+MWCNTs/GC electrode oxidation. Furthermore, square-wave voltammetry was applied to detect the sensitivity to different concentrations of agmatine at the ZnONPs+MWCNTs/GC electrode surface.

\section{RESULTS AND DISCUSSION}

Characterization of MWCNTs/GC and ZnONPs+MWCNTs/GC electrode surfaces The modified electrode surfaces were examined using SEM. The image of the MWCNTs/GC electrode surface in Figure 2A shows that the MWCNTs are homogeneously distributed on the electrode surface and that there is no significant agglutination. In addition, Figure $2 \mathrm{~B}$ shows the distribution of ZnONPs on the MWCNTs and Figure $2 \mathrm{C}$ shows the EDX data for the ZnONPs+MWCNTs/GC electrode surface. C, O, N and $\mathrm{Zn}$ were observed in the EDX analysis in Figure 2C.

\footnotetext{
surface, C) EDX analysis of ZnONPs+MWCNTs/GC electrode surface.
} 


\section{Electrochemical behavior of agmatine at the ZnONPs+MWCNTs/GC electrode surface}

Figure 3 shows the cyclic voltammograms of the agmatine molecule on the surface of a) bare/GC, b) MWCNTs/GC, c) ZnONPs/GC and d) ZnONPs+MWCNTs/GC electrodes in PBS with $50 \mathrm{mM}$ at $\mathrm{pH}$ 7.0. No peak was observed because no electrochemical response was detected for the agmatine molecule on the surface of the bare GC electrode (a). However, a broad and poor oxidation peak for the agmatine molecule was observed at $\mathrm{Epa}=+637.9 \mathrm{mV}$ on the surface of the GC electrode modified with MWCNTs (b), while a weak and clearly undefined oxidation peak for the agmatine molecule was observed at Epa $=+668.2 \mathrm{mV}$ on the surface of the GC electrode modified with ZnONPs (c). Additionally, a well-defined peak with a higher current of the agmatine molecule was observed at Epa $=+581.0 \mathrm{mV}$ on the surface of the GC electrode modified by the hybrid formation of MWCNTs and ZnONPs (d). Compared to the bare/GC, MWCNTs/GC and ZnONPs/GC electrode surfaces, the voltammetric behavior of agmatine was found to be greatly enhanced on the ZnONPs+MWCNTs/GC electrode surface with a large enhancement in the voltammetric current response. The observation of a higher current response for the agmatine molecule at the ZnONPs+MWCNTs/GC electrode surface is mainly due to the catalytic effect of the $\mathrm{ZnO}$ nanoparticles and an increase in the electron transfer rate by providing an active layer on the electrode surface (21-23). There are also studies that claim that the peak potential observed for nanoparticles of metal oxides can be attributed to the oxygen vacancies on the nanoparticles $(24,25)$. In addition, the voltammetric data indicates that agmatine oxidation is irreversible at the MWCNTs/GC and ZnONPs+MWCNTs/GC electrode surfaces due to the absence of any peak in the cathodic region.

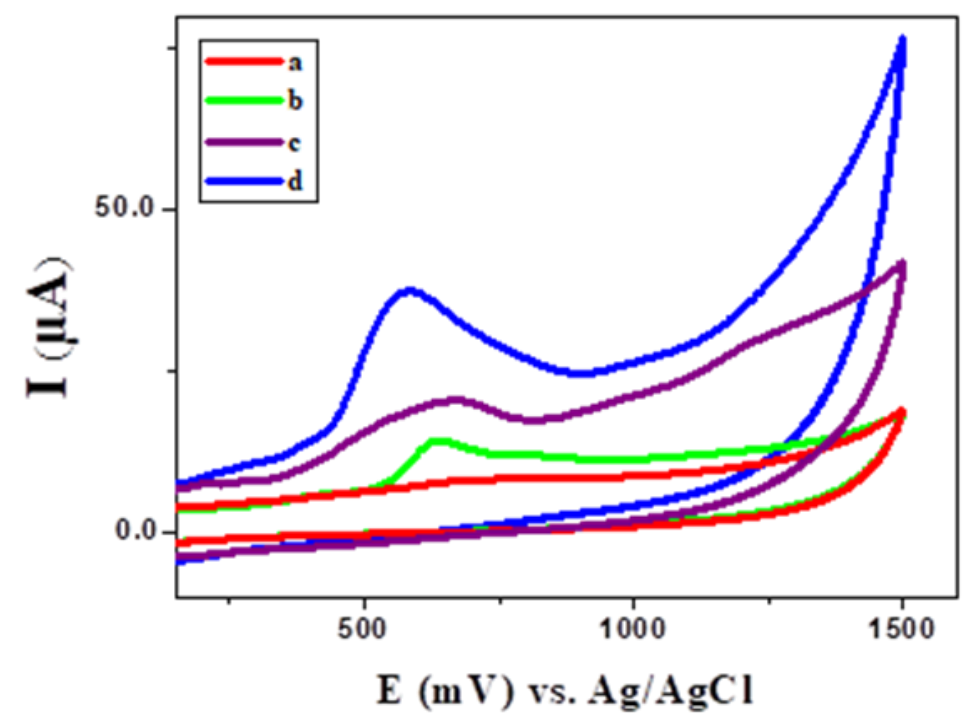

Figure 3. Cyclic voltammograms of $1.0 \times 10^{-7} \mathrm{M}$ agmatine in $50 \mathrm{mM} \mathrm{PBS}$ at $\mathrm{pH} 7.0$. at a) bare/GC, b) MWCNTs/GC, c) ZnONPs/GC, d) ZnONPs+MWCNTs/GC electrode surfaces (Scan rate: $50 \mathrm{mV} / \mathrm{s}$ ).

Figure $4 \mathrm{~A}$ shows the voltammograms at different scan rates of the agmatine solution at $1.0 \times 10^{-7} \mathrm{M}$ concentration prepared in $\mathrm{pH}$ 7.0 PBS buffer, in order to examine the effect of the scan rate on agmatine oxidation at the ZnONPs+MWCNTs/GC electrode surface. A linear curve is obtained by plotting the peak current values against the square root of the scanning speed, indicating that the reaction is diffusioncontrolled. The slope of graphic obtained by plotting the logarithm of the peak current against the logarithm of the scanning rate is close to 0.5, suggesting that electron transfer is diffusion-controlled, while a slope closer to 1.0 indicates adsorption control (26). It was determined that the graph obtained from the plot of the peak currents against the square root of the scan rate is linear $\left(R^{2}=0.9988\right)$ in Figure $4 B$, and therefore, it is thought that a diffusioncontrolled reaction occurs at the electrode surface. In Figure $4 \mathrm{C}$, the equation of the graph obtained from the logarithm of the 
peak current versus the logarithm of the scanning rate is calculated as being $y=0.4983 x-3.538$. The slope of the graph is 0.4983, indicating that the diffusion reaction is controlled. In addition, it has also been observed that agmatine peak potentials are shifted towards more anodic values (higher potentials) due to increased scanning speeds. When the agmatine peak potential shifts towards anodic values and the absence of any reduction peak are assessed together, it can be concluded that the oxidation of agmatine on the ZnONPs+MWCNTs/GC electrode surface is irreversible. Further, the behavior of the oxidation peak potential of the agmatine
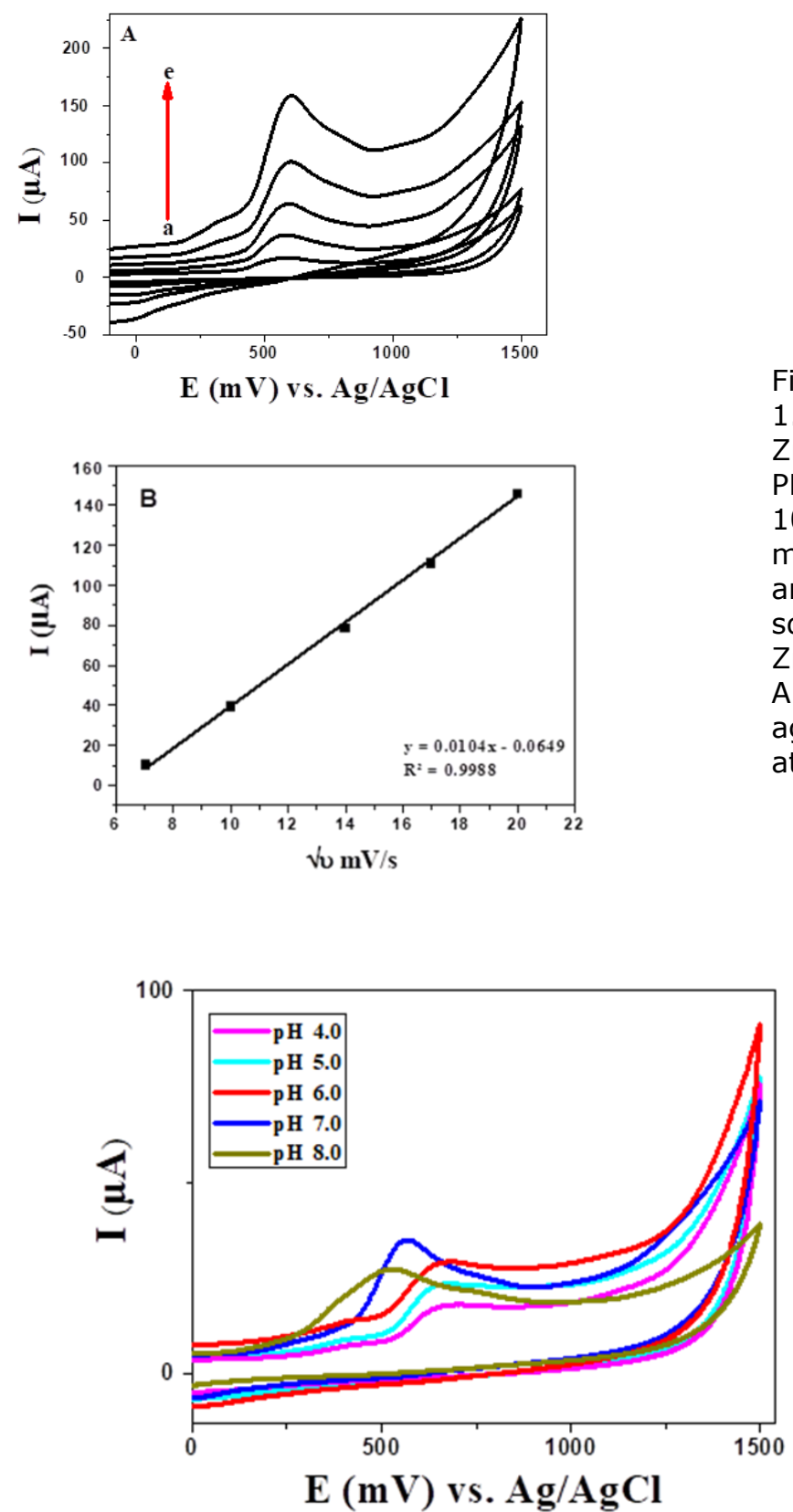

Figure 5. Cyclic voltammograms of $1.0 \times 10-7 \mathrm{M}$ agmatine at ZnONPs+MWCNTs/GC electrode in 50 $\mathrm{mM}$ PBS at $\mathrm{pH}=3.0 ; 4.0 ; 5.0 ; 6.0 ; 7.0 ; 8.0$ values. (Scan rate: $50 \mathrm{mV} / \mathrm{s}$, Equilibrium time: $5 \mathrm{~s}$ ). molecule at various $\mathrm{pH}$ values is given in Figure 5. A shift in the negative direction of the peak potential of the agmatine molecule was observed due to the increase in $\mathrm{pH}$ of the solution. This indicates that the proton is transferred during the oxidation of the agmatine molecule. Also, the voltammetric signals at $\mathrm{pH} 6.0,7.0$ and 8.0 were observed at values close to each other, as shown in Figure 5. However, since the observed voltammetric signal at $\mathrm{pH} 7.0$ has a relatively higher and smoother peak shape, this $\mathrm{pH}$ value was chosen for quantification of the agmatine molecule.

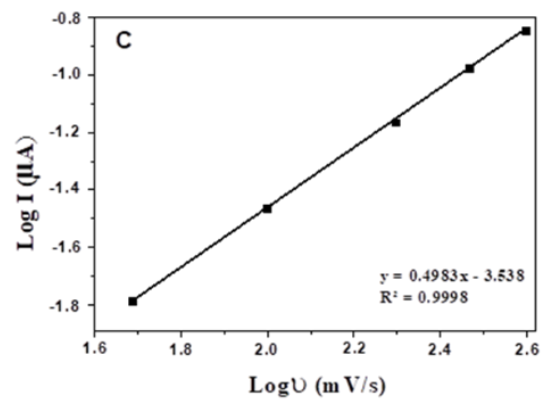

Figure 4. A) Cyclic voltammograms of 1.0 $\times 10-7 \quad M \quad$ agmatine at ZnONPs+MWCNTs/GC electrode in $50 \mathrm{mM}$ PBS at $\mathrm{pH}$ 7.0. Scan rates: a) $50 \mathrm{mV} / \mathrm{s}$; b) $100 \mathrm{mV} / \mathrm{s}$; c) 200; d) $300 \mathrm{mV} / \mathrm{s}$; e) 400 $\mathrm{mV} / \mathrm{s}$ (Equilibrium time: $5 \mathrm{~s}$ ); B) A plot of anodic peak current of agmatine versus the square root of scan rates at ZnONPs+MWCNTs/GC electrode surface; C) A plot of logarithm of anodic peak current of agmatine versus the logarithm of scan rates at ZnONPs+MWCNTs/GC electrode surface. 


\section{Quantification of agmatine}

The quantification of agmatine was performed using the SWV technique at the ZnONPs+MWCNTs/GC electrode surface. Figure $6 \mathrm{~A}$ exhibits square-wave voltammograms for different concentrations of agmatine at the ZnONPs+MWCNTs/GC electrode surface in $50 \mathrm{mM}$ PBS at pH 7.0. A linear plot was obtained with measured peak currents versus agmatine concentrations ranging from $0.1 \mu \mathrm{M}$ to $5.2 \mu \mathrm{M}$. (Figure $6 \mathrm{~B}$ ). The regression equation was calculated as $\operatorname{Ipa}(\mu \mathrm{A})=0.002 \mathrm{C}(\mu \mathrm{M})+0.0013$ with a correlation coefficient of 0.9990 . In addition, the detection limit (LOD) of the agmatine molecule was calculated as $4.13 \times 10^{-8} \mathrm{M}$ by using $\mathrm{Cm}=3 \mathrm{~S}_{\mathrm{b}} / \mathrm{m}\left(\mathrm{S}_{\mathrm{b}}\right.$ is the standard deviation of the blank signal and $\mathrm{m}$ is the slope of the regression equation).
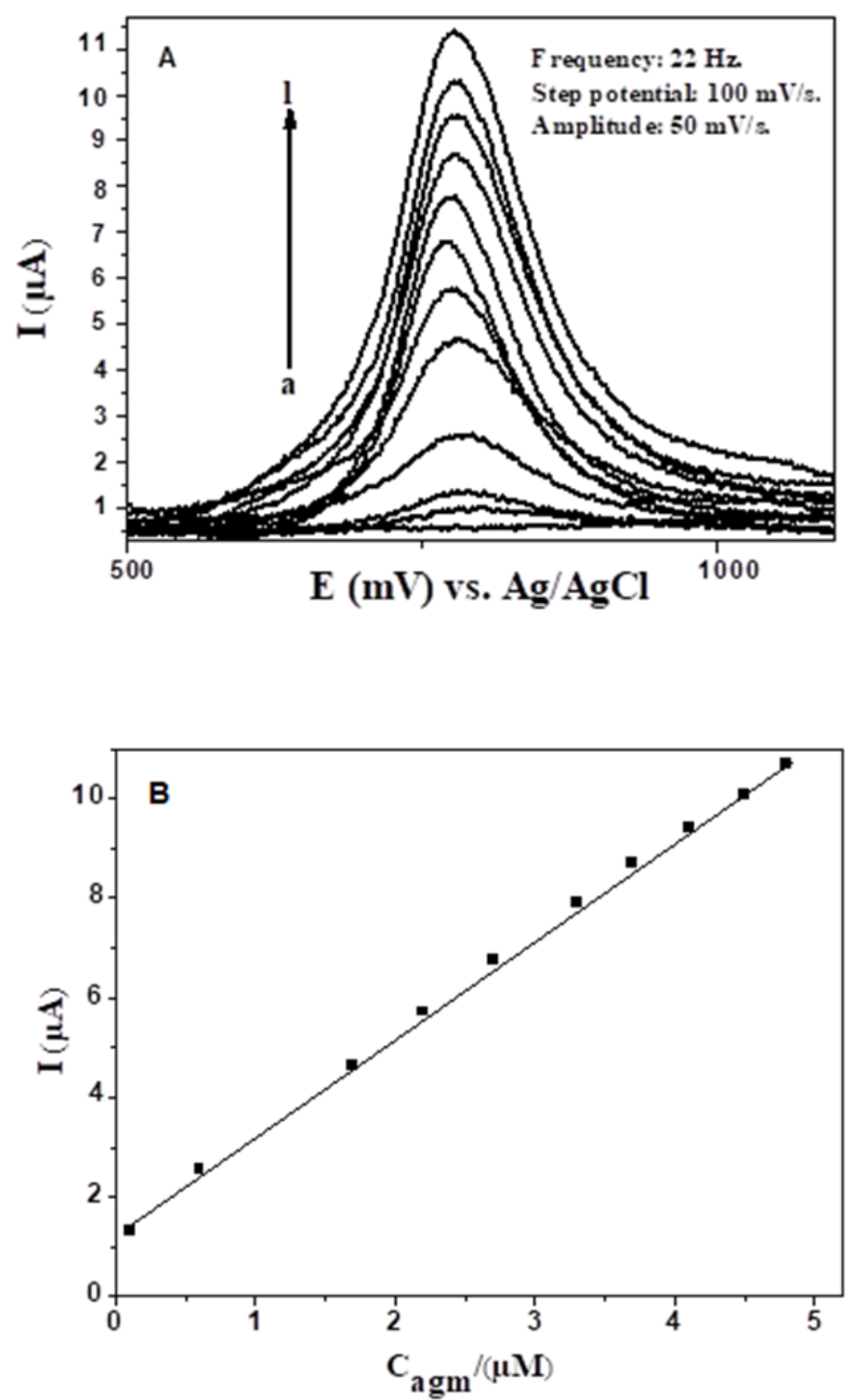

Figure 6. A) Square-wave voltammograms of various agmatine concentrations at ZnONPs+MWCNTs/GC electrode in 50 mM PBS at $\mathrm{pH}$ 7.0. Agmatine concentrations: a) $0.0 \mu \mathrm{M}$ b) $0.1 \mu \mathrm{M}$; c) $0.6 \mu \mathrm{M}$; d) $1.7 \mu \mathrm{M}$; e) $2.2 \mu \mathrm{M}$; f)
$2.7 \mu \mathrm{M}$; g) $3.3 \mu \mathrm{M}$; h) $3.7 \mu \mathrm{M}$; i) $4.1 \mu \mathrm{M}$; j) $4.5 \mu \mathrm{M}$; k) $4.8 \mu \mathrm{M}$; I) $5.2 \mu \mathrm{M}$. B) A plot of peak currents against the concentrations of agmatine.

The reproducibility of the ZnONPs+MWCNTs/GC electrode was determined by calculating the relative 
standard deviation of eight consecutive runs with the electrodes prepared in two different ways. In the first method, the electrode was redeveloped every time and voltammograms were recorded. In the second, eight different electrodes were prepared in the same way and voltammograms were recorded. The RDS of both reproducibilities for $0.2 \mu \mathrm{m}$ agmatin was calculated to be $1.8 \%$ and $1.6 \%$, respectively. This showed that the ZnONPs+MWCNTs/GC electrode had excellent reproducibility. The stability of the ZnONPs+MWCNTs/GC electrode was also examined by incubation in PBS for 40 days. Then, the voltammograms of the incubated modified electrode were recorded in $50 \mathrm{mM}$ PBS at $\mathrm{pH} 7.0$ by $\mathrm{CV}$ and compared with the voltammograms taken before incubation. Compared to those obtained before immersing the recorded voltammograms, the change in the peak current was observed to be less than $5 \%$. This slight reduction in current indicated that the ZnONPs+MWCNTs/GC electrode system had good stability.

\section{Interference study}

In order to verify the selectivity of the proposed electrode, the effects of some molecules that could make possible interference on the agmatine were also investigated by CV. For this purpose, voltammograms of the mixture containing $30 \mu \mathrm{M}$ ascorbic acid (AA), $45 \mu \mathrm{M}$ seratonin

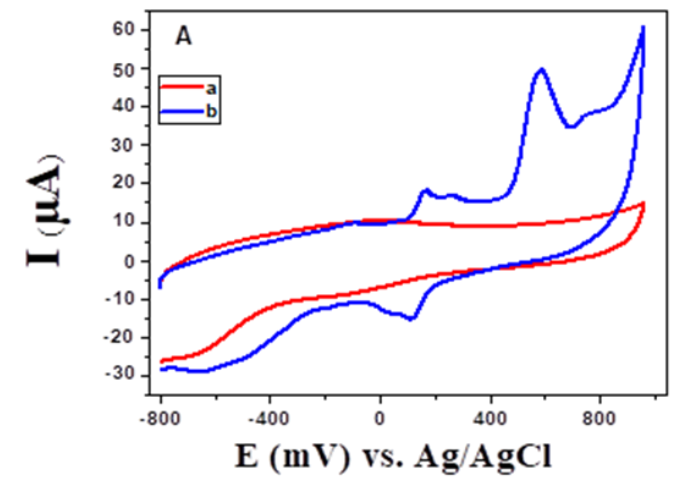

(SE) and $60 \mu \mathrm{M}$ dopamine (DA) in the presence of $0.3 \mu \mathrm{M}$ agmatine were taken on bare GC and ZnONPs+MWCNTs/GC electrodes. In Figure 7A, no peak currents of these molecules were observed on the bare GC electrode, whereas a broad and overlapping voltammetric peak current of DA and SE, a small and relatively distinct peak current of $A A$, and a clearly defined peak current of agmatine molecule were observed on the ZnONPs+MWCNTs/GC electrode. This showed that ZnO nanoparticles did not have a successful effect on the catalysis of AA, SE and DA molecules. Since AA, SE and DA have similar oxidation potentials and overlapping signals, it is already known that the detection of these species separately is a major problem on most solid electrodes (27). However, it was observed that these molecules did not have any interference effect in determining the agmatine molecule. In addition, voltamograms of increased concentrations of agmatine were also taken in the presence of $A A, S E$ and $D A$. The voltamograms showed that the increased concentration of agmatine exhibited a linear increase in anodic peak currents in Figure 7B. But there was no change in $A A, S E$ and $D A$ peak currents. The results showed that 100fold AA, 150-fold SE and 200-fold of DA molecules had no effect on the selective determination of the agmatine molecule in the proposed electrode.

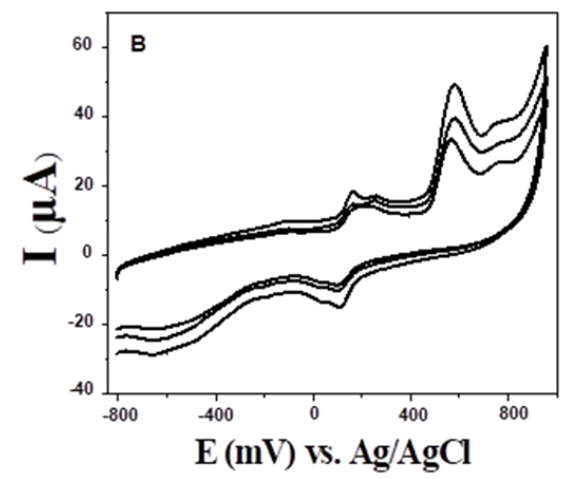

Figure 7. A) Cyclic voltammograms of $30 \mu \mathrm{M} \mathrm{AA}, 45 \mu \mathrm{M} \mathrm{SE}, 60 \mu \mathrm{M}$ DA $0.3 \mu \mathrm{M}$ agmatine in $50 \mathrm{mM}$ PBS at pH 7.0. at a) bare/GC, b) ZnONPs+MWCNTs/GC electrode surfaces (Scan rate: $50 \mathrm{mV} / \mathrm{s}$ ). B) Cyclic voltammograms of increasing concentrations of agmatine in the presence of $30 \mu \mathrm{MAA}$, $45 \mu \mathrm{M} \mathrm{SE}, 60 \mu \mathrm{M}$ DA at ZnONPs+MWCNTs/GC in in $50 \mathrm{mM}$ PBS at $\mathrm{pH}$ 7.0. (Agmatine concentrations: $0.1 \mu \mathrm{M}, 0.2 \mu \mathrm{M}, 0.3 \mu \mathrm{M}$ )

\section{CONCLUSIONS}

An electrode surface system prepared with a multi-walled carbon nanotubes (MWCNTs) hybrid decorated with ZnOPs was designed to quantify agmatine molecules quickly, economically, and reliably. The
ZnONPs+MWCNTs/GC electrode system was performed to quantifying agmatine using SWV. A linear plot of the current-concentration calibration graph in the range of $0.1 \mu \mathrm{M}$ to 5.2 $\mu \mathrm{M}$ was obtained with a detection limit of $4.13 \times 10^{-8} \mathrm{M}$. In accordance with the direction of this data, the rapid and reliable determination of the agmatine molecule, 
which causes schizophrenia and is secreted in the brain, makes this electrode system of great interest for drug development studies and clinical use.

\section{ACKNOWLEDGMENT}

This research was supported by the Nevşehir $\mathrm{Hacl}$ Bektaş Veli University Research Foundation under the project number 16F32. I would like to thank the Nevşehir Hacı Bektaş Veli University Research Foundation for their financial support.

\section{REFERENCES}

1. Singh T, Bagga N, Kaur A, Kaur N, Gawande DY, Goel RK. Agmatine for combined treatment of epilepsy, depression and cognitive impairment in chronic epileptic animals. Biomedicine \& Pharmacotherapy. 2017;92:720-5.

2. Freitas $A E$, Neis $V B$, Rodrigues ALS. Agmatine, a potential novel therapeutic strategy for depression. European Neuropsychopharmacology.

2016;26(12):1885-99.

3. Gilad GM, Salame K, Rabey JM, Gilad VH. Agmatine treatment is neuroprotective in rodent brain injury models. Life sciences. 1995;58(2):PL41-PL6.

4. Feng $\mathrm{Y}$, LeBlanc $\mathrm{MH}$, Regunathan $\mathrm{S}$. Agmatine reduces extracellular glutamate during pentylenetetrazole-induced seizures in rat brain: a potential mechanism for the anticonvulsive effects. Neuroscience letters. 2005;390(3):129-33.

5. Reis DJ, Regunathan S. Is agmatine a novel neurotransmitter in brain? Trends in pharmacological sciences. $2000 ; 21(5): 187$ 93.

6. Benítez J, García D, Romero N, González A, Martínez-Oyanedel J, Figueroa $M$, et al. Metabolic strategies for the degradation of the neuromodulator agmatine in mammals. Metabolism-Clinical and Experimental. 2018;81:35-44.

7. Bahremand T, Payandemehr P, Riazi K, Noorian AR, Payandemehr B, Sharifzadeh M, et al. Modulation of the anticonvulsant effect of swim stress by agmatine. Epilepsy \& Behavior. 2018;78:142-8.

8. Gawali NB, Bulani VD, Chowdhury AA, Deshpande PS, Nagmoti DM, Juvekar AR. Agmatine ameliorates lipopolysaccharide induced depressive-like behaviour in mice by targeting the underlying inflammatory and oxido-nitrosative mediators. Pharmacology Biochemistry and Behavior. 2016;149:1-8.

9. Uzbay TI. The pharmacological importance of agmatine in the brain. Neuroscience \& Biobehavioral Reviews. 2012;36(1):502-19.

10. Uzbay T, Goktalay G, Kayir H, Eker SS, Sarandol A, Oral $S$, et al. Increased plasma agmatine levels in patients with schizophrenia. Journal of psychiatric research. 2013;47(8):1054-60.

11. Kotagale NR, Taksande BG, Wadhwani PJ, Palhade MW, Mendhi SM, Gawande DY, et al. Psychopharmacological study of agmatine in behavioral tests of schizophrenia in rodents. Pharmacology Biochemistry and Behavior. 2012;100(3):398-403.

12. Uzbay IT, Kayir H, Göktalay G, Yildirim M. P. 3. b. 004 Agmatine induces schizophrenialike symptom in Wistar rats. European Neuropsychopharmacology. 2008;18:S399.

13. Zhang M, Wang S, Li T, Chen J, Zhu H, Du $M$. Nitrogen and gold nanoparticles co-doped carbon nanofiber hierarchical structures for efficient hydrogen evolution reactions. Electrochimica Acta. 2016;208:1-9.

14. Baytak AK, Duzmen S, Teker T, Aslanoglu $M$. Voltammetric determination of methylparaben and its DNA interaction using a novel platform based on carbon nanofibers and cobalt-nickel-palladium nanoparticles. Sensors and Actuators B: Chemical. 2017;239:330-7.

15. Wang L, Pumera M. Electrochemical catalysis at low dimensional carbons: Graphene, carbon nanotubes and beyond-A review. Applied Materials Today. 2016;5:13441.

16. Rahman MM, Ahmed J. Cd-doped Sb 204 nanostructures modified glassy carbon electrode for efficient detection of melamine by electrochemical approach. Biosensors and Bioelectronics. 2018;102:631-6.

17. Ramamoorthy C, Rajendran V. Synthesis and characterization of CuS nanostructures: Structural, optical, electrochemical and photocatalytic activity by the hydro/solvothermal process. International Journal of Hydrogen Energy. 2017;42(42):26454-63.

18. Baytak AK, Teker T, Duzmen S, Aslanoglu M. A composite material based on nanoparticles of yttrium (III) oxide for the selective and sensitive electrochemical 
determination of acetaminophen. Materials Science and Engineering: C. 2016;66:278-84.

19. Baghayeri M, Zare EN, Lakouraj MM. A simple hydrogen peroxide biosensor based on a novel electro-magnetic poly ( $p$ phenylenediamine)@ Fe3O4 nanocomposite. Biosensors and bioelectronics. 2014;55:25965.

20. Baytak AK, Teker T, Duzmen S, Aslanoglu $M$. A sensitive determination of terbutaline in pharmaceuticals and urine samples using a composite electrode based on zirconium oxide nanoparticles. Materials Science and Engineering: C. 2016;67:125-31.

21. Xu C-X, Huang K-J, Fan Y, Wu Z-W, Li J, Gan T. Simultaneous electrochemical determination of dopamine and tryptophan using a TiO2-graphene/poly (4aminobenzenesulfonic acid) composite film based platform. Materials Science and Engineering: C. 2012;32(4):969-74.

22. Baytak AK, Aslanoglu M. Voltammetric quantification of tryptophan using a MWCNT modified GCE decorated with electrochemically produced nanoparticles of nickel. Sensors and Actuators B: Chemical. 2015;220:1161-8.

23. Ye D, Luo L, Ding $Y$, Liu B, Liu $X$. Fabrication of Co 3 O 4 nanoparticlesdecorated graphene composite for determination of L-tryptophan. Analyst. 2012;137(12):2840-5.
24. Wei M-Y, Huang R, Guo L-H. High catalytic activity of indium tin oxide nanoparticle modified electrode towards electro-oxidation of ascorbic acid. Journal of Electroanalytical Chemistry. 2012;664:156-60.

25. Yang J, Lin C, Wang Z, Lin J. In $(\mathrm{OH}) 3$ and In2O3 nanorod bundles and spheres: microemulsion-mediated hydrothermal synthesis and luminescence properties. Inorganic chemistry. 2006;45(22):8973-9.

26. Sanguansak $Y$, Srimuk $P$, Krittayavathananon A, Luanwuthi S, Chinvipas $N$, Chiochan $P$, et al. Permselective properties of graphene oxide and reduced graphene oxide electrodes. Carbon. 2014;68:662-9.

27. Fayemi OE, Adekunle AS, Ebenso EE. Electrochemical determination of serotonin in urine samples based on metal oxide nanoparticles/MWCNT on modified glassy carbon electrode. Sensing and Bio-Sensing Research,2017;13: 17-27. 
